\title{
Effects of SSRI Antidepressants on Attentional Bias toward Emotional Scenes in First-Episode Depressive Patients: Evidence from an Eye-Tracking Study
}

\author{
Lei Zhang ${ }^{1,2,3,4 *}$, Fengqiong Yu', ${ }^{1,2,3,4 *}$, Qian $\mathrm{Hu}^{1,2,3,4}$, Yuxi Qiao, ${ }^{1,2,3,4}$, Rongrong Xuan ${ }^{1,2,3,4}$, \\ Gongjun $\mathrm{Ji}^{1,2,3,4}$, Chunyan Zhu ${ }^{1,2,3,4}$, Chunlan $\mathrm{Cai}^{5 凶}$, and Kai Wang ${ }^{2,3,4} \bowtie$ \\ ${ }^{1}$ Department of Medical Psychology, Chaohu Clinical Medical College, Anhui Medical University, Hefei, China \\ ${ }^{2}$ Department of Neurology, the First Affiliated Hospital of Anhui Medical University, Hefei, China \\ ${ }^{3}$ Anhui Province Key Laboratory of Cognition and Neuropsychiatric Disorders, Hefei, China \\ ${ }^{4}$ Collaborative Innovation Center for Neuropsychiatric Disorders and Mental Health, Hefei, China \\ ${ }^{5}$ Anhui Mental Health Center, Hefei, China
}

Objective Attentional biases toward emotional scenes may represent vulnerability and maintenance factors in depression. Antidepressant therapy may improve cognitive function and reduce depression, and is considered as the mechanism of action of antidepressants. Therefore, we conducted an eye-tracking test to examine whether selective serotonin re-uptake inhibitor (SSRI) antidepressants can reduce negative attentional biases and elicit clinical responses in depression.

Methods Twenty first-episode depressive patients freely viewed three types of pictures that depicted different emotional scenes (i.e., positive-control, neutral-control, and negative-control) for 4,000 ms while their eye movements were monitored. The attentional bias to different emotional scenes was assessed before and after eight weeks of SSRI treatment using the eye-tracking method. The control group included a group of healthy individuals.

Results The results revealed that first-episode depressive patients oriented their gaze more frequently to negative images and less to happy images, compared to controls. Importantly, the attentional bias in depressive patients was regulated after eight weeks of SSRI treatment. Patients showed an increased tendency to fixate on positive images and a decreased tendency to focus on negative images.

Conclusion This suggests that SSRI antidepressants decrease vulnerability to negative images, while having an effect on attention in respect to positive images.

Psychiatry Investig 2020;17(9):871-879

Key Words Attentional bias, Depression, SSRI, Emotion, Eye-tracking.

\section{INTRODUCTION}

Major depressive disorder (MDD) is a serious mental disorder, characterized by persistent low mood, lowered self-evaluation, decreased motivation, insomnia, and cognitive impair-

Received: January 2, 2020 Revised: March 17, 2020

Accepted: June 7, 2020

$\square$ Correspondence: Kai Wang, $\mathrm{PhD}$

Department of Neurology, the First Affiliated Hospital of Anhui Medical University, No.81 Meishan Road Hefei, China

Tel: +86-0551-62923704, Fax: +86-0551-62923704

E-mail: wangkai1964@126.com

$\triangle$ Correspondence: Chunlan Cai, MD

Anhui Mental Health Center, No.316 Huangshan Road, Hefei, China

Tel: +86-0551-63616116, Fax: +86-0551-63616116, E-mail: hf_ccl@126.com

*These authors contributed equally to this work.

@ This is an Open Access article distributed under the terms of the Creative Commons Attribution Non-Commercial License (https://creativecommons.org/licenses/bync/4.0) which permits unrestricted non-commercial use, distribution, and reproduction in any medium, provided the original work is properly cited. ment. ${ }^{1,2}$ At present, the general interest in MDD research focuses on the relationship between depression and cognitive deficits, ${ }^{3}$ and attentional bias is one of the core features of depression. ${ }^{4}$ According to Beck's cognitive model of depression, biased emotional processing of information plays a major role in the maintenance and development of depression. ${ }^{5}$ The schemas of depressed individuals were composed of loss, separation, failure, and worthlessness. Consequently, they exhibited a systematic bias, selectively attending and processing negative stimuli in their environment. ${ }^{6}$ It is noteworthy that recent proposals suggest that attention deployment plays a key role in mood and emotional regulation. Thus, investigations on attention-orienting biases may provide important insights into psychological vulnerability in depressive patients.

Recent studies using various tasks have shown that depression is characterized by an attentional bias towards negative 
information. Compared with healthy individuals, depressive patients showed a sustained attentional bias towards negative words that persisted throughout non-emotional processing trials. ${ }^{7}$ In addition, adolescents with depression recruit visual attention regions of threat-related stimuli to a greater extent than control adolescents. ${ }^{8}$ However, some experimental results are inconsistent. A meta-analysis of the emotional Stroop task in depression did not indicate a strong emotioncongruent bias, although emotional Stroop task has been widely used in depression research. ${ }^{9}$ Studies using the dotprobe task have found attentional biases in depression, though only under conditions of long stimuli exposures, and not under conditions involving brief durations. ${ }^{10}$ While behavioral tasks offer useful information regarding underlying attentional biases in depression, these tasks do not facilitate a thorough examination of this form of attentional processing. In each trial, response times provide one data time at the end of processing, but do not inform us about the time course of the processing, which reduces the sensitivity of this measure in terms of examining the components of attention. ${ }^{11-13}$

Eye movement recordings can be considered a useful tool to assess attentional biases to visual stimuli, allowing continuous monitoring of attentional orienting and elucidating the different components of attention. ${ }^{14,15}$ In visual tasks, eye movements are associated with attentional processes because shifts in gaze position are guided by shifts in attentional focus. ${ }^{16}$ In particular, the application of eye-tracking technology can enable researchers to measure the attentional processing of subjects directly, such as the temporal/spatial parameters of the initial orientation and the location of an initial fixation, and can provide information about the subjects' eye movements.

With selective serotonin reuptake inhibitors (SSRIs), a substantial proportion of patients with MDD respond to pharmacological treatment at week eight. ${ }^{17}$ Recent studies suggested that in depressive patients, SSRIs drugs may start to modify attentional biases in emotional information processing. ${ }^{18}$ For example, Zhou found that after eight weeks of citalopram treatment, no significant bias towards negative faces was observed in the depressive group. ${ }^{19}$ Impairments in the neutral processing of happy facial expressions in depressed individuals were evident in the core area of emotional facial processing, and were reversed following eight weeks of antidepressant treatment. ${ }^{20}$ In a neuroimaging study ${ }^{21}$ that investigated the responses of depressive patients to fearful facial expressions, those who responded to SSRIs showed a greater reduction in neutral activity across a network of regions, including the anterior cingulate, insula, amygdala, and thalamus after just seven days of escitalopram treatment. Unfortunately, there is a discrepancy between published scientific literature and successful antidepressant treatment in respect to improving cog- nitive ability, as previous reports have highlighted that poor cognition is a predictor of worsening treatment outcomes. ${ }^{17}$ Of note, Wells et al. ${ }^{22}$ found depressed participants after taking antidepressant medication demonstrated greater total gaze duration and more fixations for positive stimuli compared to non-medicated depressed participants, however, participants in the study were prescribed a variety of antidepressants (not being given a single dose). Therefore, to clearly understand the impact of depression on attention bias as a whole or its use as a treatment target, depression needs to be characterized by conducting a further attentional test using eye-tracking in both acute and SSRI antidepressant-treated states.

In accordance with previous (behavioral) findings and eyetracking evidence in MDD, we set a number of goals. First, we examined whether attentional biases are present in depressed individuals, i.e., whether untreated depressed individuals attend less to positive images or attend more to negative images than healthy controls in terms of fixation time and/or frequency. Second, we examined whether attentional biases among depressed individuals were reversed following eight weeks of antidepressant treatment, i.e., whether less attention is paid to negative emotions, while greater attention is paid to positive emotions.

\section{METHODS}

\section{Participants}

Twenty first-episode depressive patients during minor and major depressive episode were recruited from the First Affiliated Hospital of Anhui Medical University and Anhui Mental Health Center. The 20 healthy participants were recruited from local advertisements to citizens of Hefei City, Anhui Province, China. All participants were matched for age, gender, and years of education (Table 1). Two professional psychiatrists interviewed the subjects using the Chinese Revision of the Structured Clinical Interview for the DSM-IV, and consensus diagnoses were made using all available information. The following exclusion criteria: 1) subjects with other major current and past psychiatric Axis I and Axis II comorbidity such as schizophrenia, bipolar affective disorder; 2) any psychotropic drug used within 4 weeks, alcohol or substance abuse 3) history of substantial physical illness such as head trauma, neurological illness; 4) medical conditions that would contribute to psychiatric symptoms use; 5) having eye problems or difficulties in vision that were not corrected by the use of glasses or contact lenses. Exclusion criteria were the same for the healthy controls as in the patient groups. At baseline, all first-episode patients were antidepressant naive. After 2 weeks of follow-up, the patients received 100-150 mg of sertraline or 10-20 mg of escitalopram 
Table 1. Demographic data of first-episode depression patients and controls

\begin{tabular}{lccc}
\hline & First-episode depression patients $(\mathrm{N}=20)$ & Controls $(\mathrm{N}=20)$ & $\chi^{2} / \mathrm{p}$ \\
\hline Gender (male/female) & $8 / 12$ & $9 / 11$ & 0.75 \\
Age (years) & $30.5(9.9)$ & $28.8(4.4)$ & 0.64 \\
Education (years) & $12.6(2.2)$ & $14.8(2.4)$ & 0.53 \\
Hamilton depression scale score & $25.7(3.5)$ & $5.8(1.2)$ & 0.00 \\
Montreal cognitive assessment score & $27.11(0.82)$ & $27.67(0.69)$ & 0.45 \\
\hline
\end{tabular}

per day as their ongoing treatment. A clinical response to the SSRI treatment was defined as exceeding the baseline following eight weeks of treatment. ${ }^{21}$ In the patient group, cognitive status was assessed using the Montreal Cognitive Assessment $(\mathrm{MoCA})^{23}$ and the assessment of the depressive severity and treatment response was carried out using the Hamilton Depression Rating Scale (HAMD). ${ }^{24}$ Participants provided their written informed consent, and the study was approved by the Anhui Medical University Ethics Committee (20180420).

\section{Apparatus}

Gaze location and duration were measured using a remote SMI-RED eye-tracking system (SensoMotoric Instruments, Teltow, Germany), an infrared video-based eye-tracking device which allowed for recording at a frequency of $250 \mathrm{~Hz}$ (coordinates were sampled every $4 \mathrm{~ms}$ ). Visual fixations were defined as eye movements lasting more than $100 \mathrm{~ms}$ within a $1^{\circ}$ radius of visual angle. The SMI-RED Eye tracker was capable of compensating changes in head position, allowing the participant free head movements across a wide range, so that a head resting device was not required. Two Dell computers, one of which was served as the main test machine, were used to carry out the experimental operations, and the other desktop computer was used to present the experimental materials with a resolution of $1680 \times 960$. The tracker was placed beneath the desktop computer screen. Eye movements were monitored through the reflection of infrared light on the cornea and the pupil, which is sensed by the tracker (Supplementary Figures 1 and 2 in the online-only Data Supplement). Prior to the task, we performed a 9-point calibration of the participants using BeGaze software to divide the image interest area and process the gaze information in the area of interest (AOI). BeGaze software (SensoMotoric Instruments, Germany) not only provided qualitative analysis for professional screen-based eye tracking studies, such as single video analysis using gaze replay or scan paths, but also added comprehensive quantitative analytics, with key performance indicators and AOI statistics.

\section{Materials}

The stimuli included 100 pictures (i.e., 20 positive target pictures, 20 negative target pictures, 20 neutral target pictures and 40 neutral control pictures), which were selected from the International Affective Picture System (IAPS). ${ }^{25}$ Three types of emotional pictures reflected different emotional states in daily life. The happy target pictures depicted people taking pleasure in something or expressing the effect of enjoyment. Negative pictures illustrated people who were seriously threatened or hurt. Neutral pictures expressed people's daily life or emotionless activities. Control group images showed a variety of inanimate scenes. In each trial, one emotional picture (i.e., positive or neutral or negative) and one control picture were presented together. The order of each trial involving two pictures was randomized, producing three types of emotional trials, i.e., happy-control, negative-control, and neutral-control trials.

Each experiment began with a fixed cross displayed centrally on a 22-inch monitoring computer screen (screen resolution: $1920 \times 1080$ pixels), followed by a pair of two pictures, which were presented for 4,000 ms. Participants were requested to view pairs of pictures freely while recording their eye movements. After 4,000 ms of viewing the picture pairs, the pictures disappeared and the fixed cross reappeared at the same time, which indicated the commencement of the next trail. Before the formal experiment, participants were shown the task with six practical trials involving emotional and control pictures. Thus, a total of 80 trials $(60$ study +20 filler) were presented. Twenty filler trials with two neutral control images were presented to obscure the nature of the task. The experimental design is presented in Figure 1.

\section{Design and procedure}

The study employed a case-controlled observational experiment. The sample of first-episode depressive patients $(n=20)$ and healthy controls $(n=20)$ were assessed at baseline using HAMD, MoCA, and an eye movement experiment. Patients were administered SSRIs each morning for a period of eight weeks without an adjustment of the dosage. In respect to the depressive patients group, assessments of attentional bias and treatment response were conducted using the eye-tracking method at baseline, as well as at week eight. Healthy controls included 20 participants who were assessed at baseline only. 


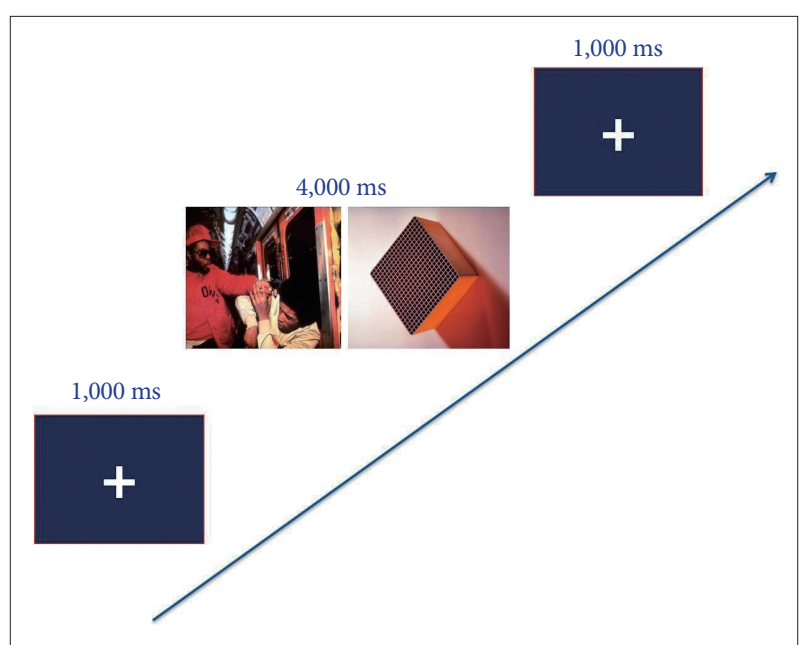

Figure 1. Examples of one negative target picture and one control picture in a trial.

\section{Data analyses}

In the free-viewing experiment, we computed the following eye movement indicators to reflect the participants' attention to the pictures: 1 ) total fixation time (the total duration of the gaze in response to emotional pictures during the $4,000 \mathrm{~ms}$ free-viewing period); 2) total number of fixations (the total number of times that each participant fixed and re-fixed on a specific target picture); 3) mean glance duration (average time of each participant's gaze was maintained within a specific boundary of a target image, i.e., the period of time between when the gaze focused on the image and when the gaze focused away from the image); 4) first-pass fixation duration (the sum of fixation duration of the image when it is first viewed) To investigate group differences in attentional bias at baseline, a $2 \times 3$ repeated-measures ANOVA was conducted, with the between-subject factor group (first-episode depression patient group at baseline, control group), and valence (positive, neutral, negative) as within-subjects factors for each eye-tracking indicator (i.e., first-pass fixation duration, total fixation number, total fixation time, and glance duration). To evaluate the therapeutic effects of SSRIs on attentional bias, first, a $2 \times 3$ repeated-measure ANOVA was conducted, with the within-subjects factors drug (baseline, eight weeks) and valence (positive, neutral, negative) in the patient group. When the interaction was significant, a paired-samples t-test was carried out to perform planned comparisons, which consisted in within-group comparisons before and after eight weeks of medication treatment. Second, we conducted a $2 \times 3$ repeated-measures ANOVA to examine the effect of SSRI treatment, with the between-subject factor group (first-episode patient group after eight weeks of treatment, control group), valence (positive, neutral, negative) as within-subjects factors for each eye-tracking indicator.

\section{RESULTS}

\section{Demographic characteristics}

Demographic characteristics for participants according to the levels of depression, cognitive function, gender, age, and education are presented in Table 1. The statistical analyses did not reveal any significant differences in gender, age, and education between the two groups.

\section{Attentional bias}

\section{Total number of fixations}

The ANOVA of the total number of fixations revealed the effect of valence $[\mathrm{F}(1,38)=5.82, \mathrm{p}=0.008]$ and the effect of group $[\mathrm{F}(1,38)=6.64, \mathrm{p}=0.014]$, which was qualified by an interaction of group $\times$ valence $[F(2,76)=15.40, p<0.001]$. The simple effect tests on the effects of the groups for each valence revealed significant differences between the groups for the positive pictures $(\mathrm{t}=-4.73, \mathrm{p}<0.001)$ and negative pictures $(\mathrm{t}=2.18, \mathrm{p}=0.035)$ (Figure 2A).

\section{Total fixation time}

The ANOVA of the total fixation time revealed an effect of group $[\mathrm{F}(1,38)=5.72, \mathrm{p}=0.02]$. Importantly, we found a significant group $\times$ valence interaction $[F(2,76)=27.60, p<0.001]$. A simple effect analysis of the total fixation time of different emotional pictures revealed statistical difference for positive pictures between the two groups ( $\mathrm{t}=-5.09, \mathrm{p}<0.001)$, suggesting that depressive patients paid less attention to positive emotions compared to the control group (Figure 2B).

\section{Mean glance duration}

The analyses showed a significant valence $\times$ group interaction, $[\mathrm{F}(2,76)=27.97, \mathrm{p}<0.001]$. Regarding differences between groups, simple effect tests revealed that, in comparison with the control group, patients showed a higher tendency to fixate on negative pictures $(t=4.06, p<0.001)$ and lower tendency to fixate on positive pictures $(\mathrm{t}=-4.30, \mathrm{p}<0.001)$ (Figure $2 \mathrm{C}$ ).

\section{First-pass fixation duration}

The ANOVA of the first-pass fixation duration revealed a non-significant effect for group $\times$ valence $[\mathrm{F}(2,76)=1.88$, $\mathrm{p}>0.05$ ] (Table 2).

\section{SSRIs effects on attentional bias}

\section{Total number of fixations}

A $2 \times 3$ repeated-measures ANOVA was conducted to assess the effects of SSRIs on the total number of fixations. A significant within-subjects interaction of drug and valence 
was found $[\mathrm{F}(2,38)=8.95, \mathrm{p}=0.003]$. A paired-samples $\mathrm{t}$-test revealed that more number of fixations $(\mathrm{t}=-2.46, \mathrm{p}=0.024)$ for positive pictures, while less number of fixations was found for negative pictures $(t=1.98, p=0.048)$ after eight weeks of SSRI treatment in the patient group (Figure 3A). There was a non-significant interaction between group and
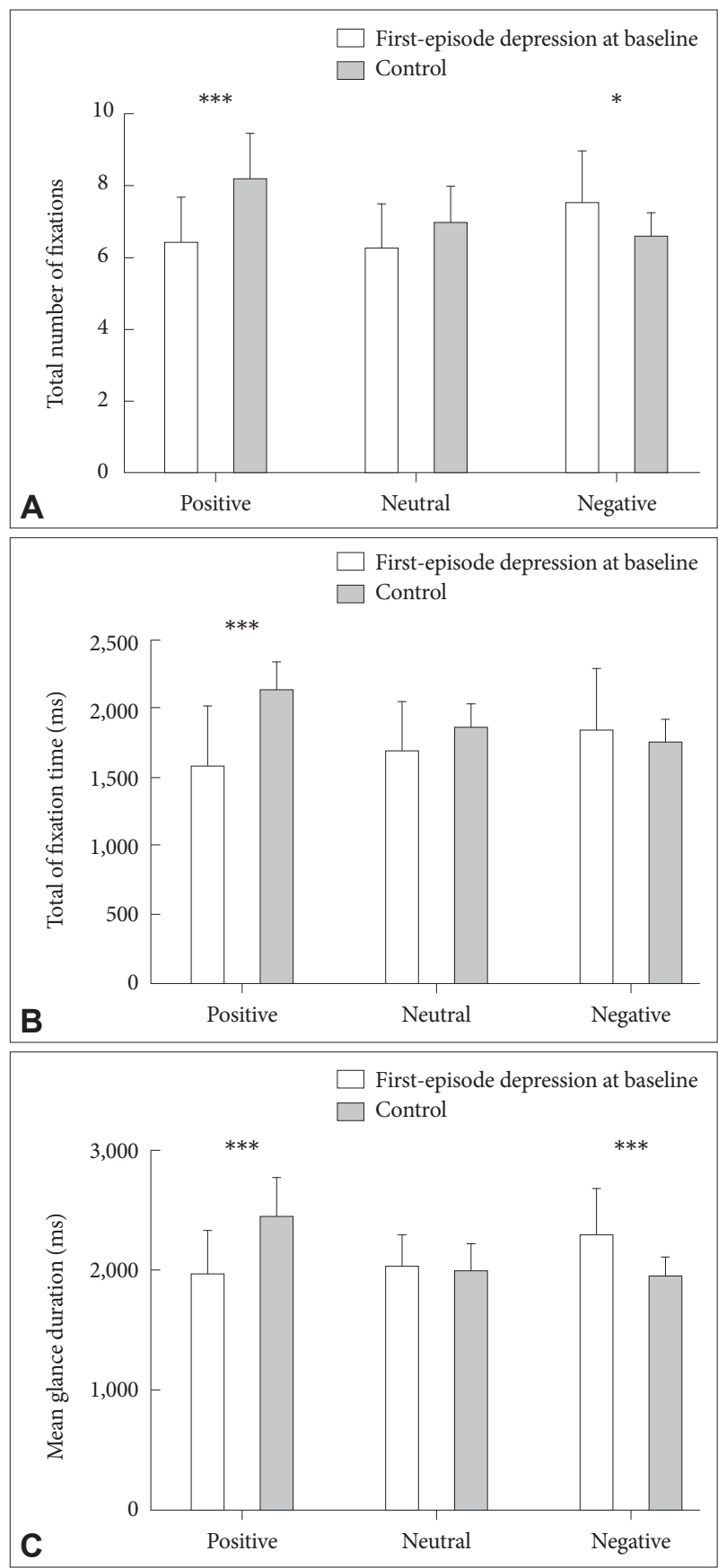

Figure 2. A: total number of fixations on each valence for pretreatment depressed patientes and control groups. B: total fixation time on each valence for pre-treatment depressed patients and control groups. C: mean glance duration on each valence for pre-treatment depressed patients and control groups. Error bars indicate standard error. ${ }^{*} \mathrm{p}<0.05,{ }^{* * *} \mathrm{p}<0.001$. valence $[\mathrm{F}(2,76)=0.69, \mathrm{p}>0.05]$. These results indicated that treatment with SSRIs significantly adjusted the attentional bias of depressive patients.

\section{Total fixation time}

The ANOVA of the effects of SSRIs in respect to changing attentional bias showed that the interaction of valence $\times$ drug was significant $[\mathrm{F}(2,38)=14.47, \mathrm{p}<0.001]$. Following SSRI therapy (eight weeks), the fixation time for positive targets was markedly increased (i.e., paired difference between preand post-treatment: $\mathrm{t}=-3.26, \mathrm{p}=0.004$ ) (Figure $3 \mathrm{~B}$ ). In addition, a $2 \times 3$ repeated-measures ANOVA was conducted, with the between-subject factor group, and valence as within-subjects factors for the effect following eight weeks of medication treatment. Neither the effect of group nor the interaction between valence and group approached significance [F (1, $38)=2.34, \mathrm{p}>0.05 ; \mathrm{F}(2,76)=0.59, \mathrm{p}>0.05]$. This result indicated that attention to positive pictures in medicated depressive patients was close to "normal" when compared to controls.

\section{Mean glance duration}

The ANOVA results revealed that the interaction of valence $\times$ drug was significant $[F(2,38)=18.29, \mathrm{p}<0.001]$. To explore this difference in the patient group, a paired-samples t-test was carried out, revealing a longer fixation time for positive pictures $(\mathrm{t}=-4.33, \mathrm{p}=0.015)$, while a shorter fixation time was found for negative pictures $(t=2.81, \mathrm{p}=0.01$ ) (Figure $3 \mathrm{C}$ ). Furthermore, the results of the analyses showed a non-significant valence $\times$ group interaction after eight weeks of SSRI treatment, $[\mathrm{F}(2,76)=1.78, \mathrm{p}>0.05]$.

\section{First-pass fixation duration}

The interaction of valence $\times$ drug was not significant between pre- and post-treatment in the depressive patients group $[\mathrm{F}(2,38)=2.12, \mathrm{p}>0.05]$ (Table 2$)$.

\section{Correlations of eye-tracking variables with HAMD scores}

We also examined the relationship between eye-tracking parameters and clinical severity, which was measured using HAMD. Specifically, the bias scores for the negative images (i.e., the total number of fixations and glance duration) were significantly correlated with the participants' mood symptoms $(\mathrm{r}=0.35,0.39 ; \mathrm{p}=0.039,0.044)$. In addition, depressive symptoms were associated with the happy images bias in respect to the total fixation time and glance duration $(r=-0.36$, $-0.43 ; \mathrm{p}=0.03,0.009)$. This is consistent with the idea that clinical severity plays a major role in the emotion-related attentional biases in depressed individuals. 
Table 2. Mean and standard deviation for the total fixation time, the glance duration, the total number of fixations and the first-pass fixation duration for each stimulus category for depression at baseline, depression after 8 weeks treatment and control groups

\begin{tabular}{|c|c|c|c|c|c|c|}
\hline \multirow{2}{*}{ Stimulus category } & \multicolumn{2}{|c|}{ Depression at baseline $(\mathrm{N}=20)$} & \multicolumn{2}{|c|}{ Depression after 8 weeks treatment $(\mathrm{N}=20)$} & \multicolumn{2}{|c|}{ Controls $(\mathrm{N}=20)$} \\
\hline & M & SD & $\mathrm{M}$ & SD & M & $\mathrm{SD}$ \\
\hline \multicolumn{7}{|c|}{ Total fixation time (ms) } \\
\hline Positive & 1,584 & 410 & 1,912 & 475 & 2,112 & 215 \\
\hline Neutral & 1,669 & 356 & 1,718 & 433 & 1,853 & 149 \\
\hline Negative & 1,839 & 423 & 1,636 & 450 & 1,731 & 167 \\
\hline \multicolumn{7}{|l|}{ Glance duration (ms) } \\
\hline Positive & 1,939 & 356 & 2,329 & 379 & 2,402 & 323 \\
\hline Neutral & 2,006 & 246 & 2,086 & 295 & 1,971 & 235 \\
\hline Negative & 2,284 & 350 & 1,970 & 334 & 1,941 & 153 \\
\hline \multicolumn{7}{|c|}{ Total number of fixations } \\
\hline Positive & 6.25 & 1.21 & 7.24 & 1.48 & 8.02 & 1.14 \\
\hline Neutral & 6.14 & 1.18 & 6.29 & 1.16 & 6.88 & 0.96 \\
\hline Negative & 7.23 & 1.38 & 6.50 & 1.10 & 6.82 & 0.69 \\
\hline \multicolumn{7}{|c|}{ First-pass fixation duration (ms) } \\
\hline Positive & 254 & 69 & 290 & 123 & 268 & 99 \\
\hline Neutral & 234 & 74 & 276 & 170 & 281 & 145 \\
\hline Negative & 278 & 105 & 271 & 147 & 275 & 98 \\
\hline
\end{tabular}

$\mathrm{N}$ : number, M: mean, SD: standard deviation, ms: millisecond

\section{DISCUSSION}

The current study examined the effects of SSRIs on attentional bias in depressive patients by carrying out a free-viewing eye-tracking experiment. The key findings can be summarized as follows. First, the initial orientation of all participants (both patients and healthy controls) was determined: First-episode depressive patients oriented their gaze more frequently to negative images and less to happy images, compared to controls. Second, the attentional bias in depressive patients was regulated following eight weeks of SSRI treatment. Patients showed an increased tendency to fixate on positive pictures and a decreased tendency to fixate on negative pictures. In the SSRImedicated depressive patients, attention to emotional pictures (positive and negative) was close to "normal" when compared to controls. Furthermore, the attentional biases of depressive patients were associated with clinical severity.

In respect to the nature of emotional biases in depressive patients, the biases toward positive and negative images were state-dependent. On the one hand, comparable to patients with bipolar disorder in the depressive phase ${ }^{26}$ depressive patients paid less attention to the positive images than the control group, as deduced from the total fixation time, total number of fixations, and glance duration found for these images. On the other hand, in comparison to healthy controls, depressive patients paid more attention to negative images in terms of the total number of fixations and glance duration, and this result was similar to previous eye-tracking studies with individuals with major depressive disorder. ${ }^{27-29}$ According to Beck's cognitive vulnerability-stress theories, ${ }^{5}$ dysfunctional cognitive structures in individuals with depression may condition the attentional preferences and constitute a general vulnerability factor. This negative attentional bias toward threatening information could increase the emotional reactivity of depressive patients, and may contribute to the onset or exacerbation of an affective episode when people with depression experience stressful life events. In depressive patients, the high emotional reactivity to stressful events, influenced by a hypervigilance to threatening stimuli, affected the formation and continued progression of depression. ${ }^{5,30}$

Previous studies found that serotonin plays an important role in the processing of affective stimuli and inhibitory control of behavior and adaptation, ${ }^{31}$ and in healthy volunteers, lowering brain serotonin level results in increased sensitivity to punishment and negative feedback without affecting reward. ${ }^{32,33}$ These results highlight that in depressive patients, a decrease in serotonin concentration could lead to an attentional bias toward negative stimuli and away from positive stimuli. ${ }^{34,35} \mathrm{~A}$ study found that depressive patients who were administered SSRIs received significantly less information from negative feedback than medication naive depressive patients, which indicated that SSRI antidepressants diminish the capacity to learn from negative feedback. ${ }^{36}$ Similar to our findings, following eight weeks of escitalopram treatment, no significant attentional bias toward 
negative faces was observed in depressive patients, using a dot probe task of facial expressions. ${ }^{19} \mathrm{~A}$ body of research results suggest that these alterations in emotional processing highlight the effectiveness of SSRIs in acting on a neutral modulation circuit, which is strongly innervated by serotonin, namely, the limbic and prefrontal cortex (PFC) ${ }^{37,38}$

Recent imaging studies argued that depressive patients who
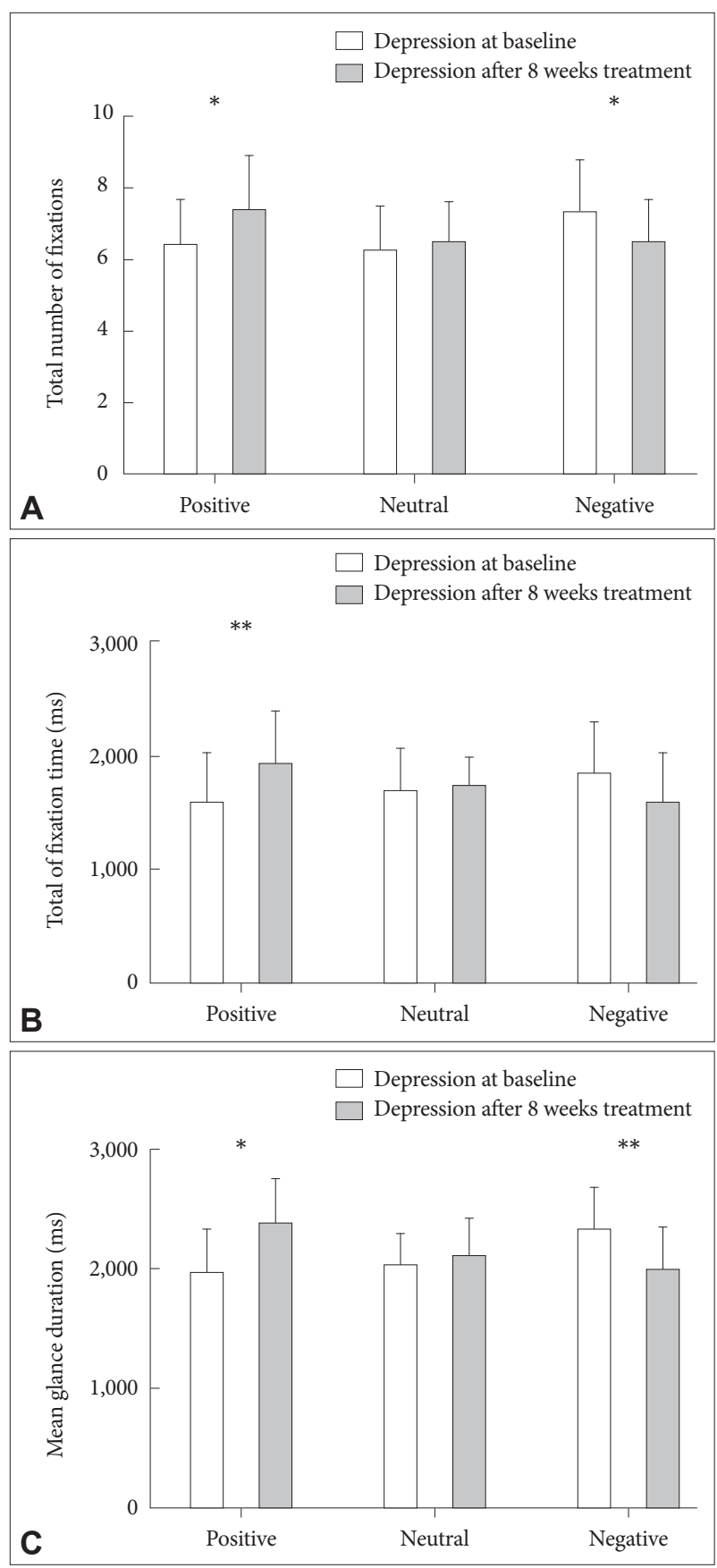

Figure 3. A: Total number of fixations on each valence for pre- and post-treatment depressed patients. B: Total fixation time on each valence for pre- and post-treatment depressed patients. C: Mean glance duration on each valence for pre- and post-treatment depressed patients. ${ }^{*} p<0.05,{ }^{* *} p<0.01$. went on to respond to SSRIs experienced a greater decrease in neutral activity, observed across a network of regions, including the anterior cingulated and amygdale, ${ }^{39,40}$ in response to facial expressions of fear and pleasure after just seven days of escitalopram treatment. ${ }^{21}$ Short-term administration of SSRIs can regulate PFC responses to negative self-reference stimuli in individuals at a high risk of depression. ${ }^{41}$ Therefore, early modification of negative bias may play a key role in the drug action of SSRIs and is a potential predictor of therapeutic response. In addition, the drug action of SSRIs normalizes the blood-oxygen-level-dependent (BOLD) response in the PFC and the functional connection between PFC and amygdala. ${ }^{36}$ The PFC and limbic-cortical regions constitute the key nodes of depression models, and functional neuroimaging studies have identified these regions as the basis of negative bias on emotional processing in depressive patients. ${ }^{42}$ The above results show that antidepressants modulate emotional processing, increasing positive emotional attention and reducing negative attentional bias.

In a previous study using a dot-probe task, ${ }^{43}$ we demonstrated that attentional biases in the processing of emotional facial expressions are evident (e.g., participants selectively attended to sad faces, which indicated a positive bias that was not observed) in remitted depressed patients. This investigation of an attentional bias in formerly depressed participants suggested that cognitive biases are stable beyond the depressive episode. In contrast, our results showed an increased tendency to fixate on positive pictures and a decreased tendency to fixate on negative pictures in treated depressed patients. This may be partially due to the difference in the depression status in the former study and the present study. In the former study, remitted depressive patients were selected to examine the attentional bias and were compared to controls. It is possible that sad expressions capture attention in remitted depressive patients who are concerned about becoming depressed again in the future because such negative stimuli relate to their own concerns. In addition, in the former study, a behavioral experiment (dot-probe task) was conducted to examine the attentional bias between remitted depressive patients and controls, whereas a free-viewing eye-tracking task was used to directly investigate the difference in attentional function in treated depressive patients and controls. Although behavioral experiments offer useful information on underlying emotional processing, there is an inherent limitation in respect to response times. Response times from behavioral experiments do not inform us about the time course of the underlying attentional processes. However, eye movements are related to attentional processes during visual tasks and provide an online measure of emotional processing.

Our study illuminates the relationship between attentional 
bias and clinical severity in depression. Consistent with the several prior studies, ${ }^{44,45}$ severity of depressive symptoms was positively associated with negative images bias, in contrary, negatively associated with happy images bias. Interesting, Wells et al. ${ }^{46}$ found that attention training could lead to a reduction in depressive symptoms while subjects in the no training condition experienced relatively little change. The results of this study bolster the argument that, selective attention bias for negative images plays a key role in the maintenance of depressive symptoms.

From the point of view of the relevant statistical data, after 8 weeks of treatment, total fixation time on negative image in depression showed a declining trend. However, the difference is not statistically significant. A major limitation of the current study is the relatively small sample size of 20 first-episode depressive patients, so the research results should be considered as strictly. Another limitation relates to the fact that the attentional bias in depressive patients was regulated after SSRI treatment at the eye-tracking level. Further investigations should employ a wide range of experimental tasks and designs, as well as recording methodologies in order to facilitate a more comprehensive understanding of brain networks underlying the relationship between depression and attentional processing.

In summary, our study demonstrated that first-episode depressive patients oriented their gaze more frequently to negative images and less to happy images, compared to controls. Importantly, the present study provides key evidence that following eight weeks of antidepressant treatment, the attentional bias changes in first-episode depressive patients, who showed an increasing tendency to fixate on positive pictures and a decreasing tendency to fixate on negative pictures. These observations may be relevant for the development of improved therapeutic approaches to depression.

\section{Supplementary Materials}

The online-only Data Supplement is available with this article at https://doi.org/10.30773/pi.2019.0345.

\section{Acknowledgments}

This research was supported by the Natural Science Foundation of China (91432301, 81771456, 81971689, 31771222 and 31571149), the Natural Science Research Project of Anhui Province (KJ2019A0239), the Humanities and Social Sciences Project of Anhui Province (SK2019A0177).

\section{Conflicts of Interest}

The authors have no potential conflicts of interest to disclose.

\section{Author Contributions}

Conceptualization: Kai Wang, Chunlan Cai. Data curation: Lei Zhang, Rongrong Xuan. Formal analysis: Fengqiong Yu, Qian Hu, Yuxi Qiao. Funding acquisition: Kai Wang. Methodology: Lei Zhang, Fengqiong Yu. Project administration: Gongjun Ji, Chunyan Zhu. Resources: Chunlan
Cai. Software: Kai Wang. Supervision: Kai Wang, Chunlan Cai. Validation: Lei Zhang, Qian Hu. Writing_original draft: Lei Zhang, Fengqiong Yu. Writing_review \& editing: Kai Wang, Chunlan Cai.

\section{ORCID iDs}

$\begin{array}{ll}\text { Lei Zhang } & \text { https://orcid.org/0000-0002-5458-8618 } \\ \text { Fengqiong Yu } & \text { https://orcid.org/0000-0002-6480-4740 } \\ \text { Qian Hu } & \text { https://orcid.org/0000-0003-4953-337X } \\ \text { Yuxi Qiao } & \text { https://orcid.org/0000-0002-2453-239X } \\ \text { Rongrong Xuan } & \text { https://orcid.org/0000-0001-6590-4199 } \\ \text { Gongjun Ji } & \text { https://orcid.org/0000-0002-7073-5534 } \\ \text { Chunyan Zhu } & \text { https://orcid.org/0000-0002-3434-2581 } \\ \text { Chunlan Cai } & \text { https://orcid.org/0000-0002-1748-131X } \\ \text { Kai Wang } & \text { https://orcid.org/0000-0002-6197-914X }\end{array}$

\section{REFERENCES}

1. Mojtabai R, Olfson M, Han B. National trends in the prevalence and treatment of depression in adolescents and young adults. Pediatrics 2016;138:e20161878.

2. Afridi MI, Hina M, Qureshi IS, Hussain M. Cognitive disturbance comparison among drug-naive depressed cases and healthy controls. J Coll Physicians Surg Pak 2011;21:351-355.

3. Steffens DC, Otey E, Alexopoulos GS, Butters MA, Cuthbert B, Ganguli M, et al. Perspectives on depression, mild cognitive impairment, and cognitive decline. Arch Gen Psychiatry 2006;63:130-138.

4. Rock PL, Roiser JP, Riedel WJ, Blackwell AD. Cognitive impairment in depression: a systematic review and meta-analysis. Psychol Med 2014; 44:2029-2040.

5. Beck AT. The evolution of the cognitive model of depression and its neurobiological correlates. Am J Psychiatry 2008;165:969-977.

6. Posne MI, Rothbart MK. Developing mechanisms of self-regulation. Dev Psychopathol 2000;12:427-441.

7. Fritzsche A, Dahme B, Gotlib IH, Joormann J, Magnussen H, Watz H, et al. Specificity of cognitive biases in patients with current depression and remitted depression and in patients with asthma. Psychol Med 2010;40:815-826.

8. Colich NL, Ho TC, Foland-Ross LC, Eggleston C, Ordaz SJ, Singh $\mathrm{MK}$, et al. Hyperactivation in cognitive control and visual attention brain regions during emotional interference in adolescent depression. Biol Psychiatry Cogn Neurosci Neuroimaging 2017;2:388-395.

9. Epp AM, Dobson KS, Dozois DJ, Frewen PA. A systematic meta-analysis of the Stroop task in depression. Clin Psychol Rev 2012;32:316-328.

10. Sanchez A, Vazquez C, Marker C, LeMoult J, Joormann J. Attentional disengagement predicts stress recovery in depression: an eye-tracking study. J Abnorm Psychol 2013;122:303-313.

11. Vazquez C, Blanco I, Sanchez A, Mcnally RJ. Attentional bias modification in depression through gaze contingencies and regulatory control using a new eye-tracking intervention paradigm: study protocol for a placebo-controlled trial. BMC Psychiatry 2016;16:439.

12. Waechter S, Nelson A, Wright C, Hyatt A, Oakman J. Measuring attentional bias to threat: reliability of dot probe and eye movement indices. Cogn Ther Res 2014;38:313-333.

13. Victor TA, Furey ML, Fromm SJ, Ohman A, Drevets WC. Relationship between amygdala responses to masked faces and mood state and treatment in major depressive disorder. Arch Gen Psychiatry 2010;67:11281138.

14. Amor TA, Reis SD, Campos D, Herrmann HJ, Andrade JS Jr. Persistence in eye movement during visual search. Sci Rep 2016;6:20815.

15. Hermans D, Vansteenwegen D, Eelen P. Eye movement registration as a continuous index of attention deployment: data from a group of spider anxious students. Cogn Emot 1999;13:419-434.

16. Rayner K. Eye movements and attention in reading, scene perception, and visual search. Q J Exp Psychol (Hove) 2009;62:1457-1506.

17. Shilyansky C, Williams LM, Gyurak A, Harris A, Usherwood T, Etkin 
A. Effect of antidepressant treatment on cognitive impairments associated with depression: a randomised longitudinal study. Lancet Psychiatry 2016;3:425-435.

18. Rosenberg C, Lauritzen L, Brix J, Jorgensen JB, Kofod P, Bayer LB. Citalopram versus amitriptyline in elderly depressed patients with or without mild cognitive dysfunction: a danish multicentre trial in general practice. Psychopharmacol Bull 2007;40:63-73.

19. Zhou Z, Cao S, Li H, Li Y. Treatment with escitalopram improves the attentional bias toward negative facial expressions in patients with major depressive disorders. J Clin Neurosci 2015;22:1609-1613.

20. Fu CH, Williams SC, Brammer MJ, Suckling J, Kim J, Cleare AJ, et al. Neural responses to happy facial expressions in major depression following antidepressant treatment. Am J Psychiatry 2007;164:599-607.

21. Godlewska BR, Browning M, Norbury R, Cowen PJ, Harmer CJ. Early changes in emotional processing as a marker of clinical response to SSRI treatment in depression. Transl Psychiatry 2016;6:e957.

22. Wells TT, Clerkin EM, Ellis AJ, Beevers CG. Effect of antidepressant medication use on emotional information processing in major depression. Am J Psychiatry 2014;171:195-200.

23. O'Driscoll C, Shaikh M. Cross-cultural applicability of the Montreal Cognitive Assessment (MoCA): a systematic review. J Alzheimers Dis 2017;58:789-801.

24. Hamilton M. A rating scale for depression. J Neurol Neurosurgery 1960;23:56-62.

25. Gruhn D, Scheibe S. Age-related differences in valence and arousal ratings of pictures from the International Affective Picture System (IAPS): do ratings become more extreme with age? Behav Res Methods 2008;40:512-521.

26. Garcia-Blanco A, Salmeron L, Perea M. Attentional capture by emotional scenes across episodes in bipolar disorder: evidence from a freeviewing task. Biol Psychol 2015;108:36-42.

27. Leyman L, De Raedt R, Vaeyens R, Philippaerts RM. Attention for emotional facial expressions in dysphoria: an eye-movement registration study. Cogn Emot 2011;25:111-120.

28. Eizenman M, Yu LH, Grupp L, Eizenman E, Ellenbogen M, Gemar M, et al. A naturalistic visual scanning approach to assess selective attention in major depressive disorder. Psychiatry Res 2003;118:117-128.

29. Kellough JL, Beevers CG, Ellis AJ, Wells TT. Time course of selective attention in clinically depressed young adults: an eye tracking study. Behav Res Ther 2008;46:1238-1243.

30. Disner SG, Beevers CG, Haigh EA, Beck AT. Neural mechanisms of the cognitive model of depression. Nat Rev Neurosci 2011;12:467-477.

31. Cha J, Guffanti G, Gingrich J, Talati A, Wickramaratne P, Weissman M, et al. Effects of serotonin transporter gene variation on impulsivity mediated by default mode network: a family study of depression. Cereb Cortex 2018;28:1911-1921.
32. Robinson OJ, Cools R, Sahakian BJ. Tryptophan depletion disinhibits punishment but not reward prediction: implications for resilience. Psychopharmacology (Berl) 2012;219:599-605.

33. Cools R, Robinson OJ, Sahakian B. Acute tryptophan depletion in healthy volunteers enhances punishment prediction but does not affect reward prediction. Neuropsychopharmacology 2008;33:2291-2299.

34. Hommer RE, Meyer A, Stoddard J, Connolly ME, Mogg K, Bradley $\mathrm{BP}$, et al. Attention bias to threat faces in severe mood dysregulation. Depress Anxiety 2014;31:559-565.

35. Jenness JL, Young JF, Hankin BL. 5-HTTLPR moderates the association between attention away from angry faces and prospective depression among youth. J Psychiatr Res 2017;91:83-89.

36. Herzallah MM, Moustafa AA, Natsheh JY, Abdellatif SM, Taha MB, Tayem YI, et al. Learning from negative feedback in patients with major depressive disorder is attenuated by SSRI antidepressants. Front Integr Neurosci 2013;7:67.

37. Clark DA, Beck AT. Cognitive theory and therapy of anxiety and depression: convergence with neurobiological findings. Trends Cogn Sci 2010;14:418-424.

38. Price JL, Drevets WC. Neurocircuitry of mood disorders. Neuropsychopharmacology 2010;35:192-216.

39. Fu CH, Williams SC, Cleare AJ, Brammer MJ, Walsh ND, Kim J, et al. Attenuation of the neural response to sad faces in major depression by antidepressant treatment: a prospective, event-related functional magnetic resonance imaging study. Arch Gen Psychiatry 2004;61:877-889.

40. Sheline YI, Barch DM, Donnelly JM, Ollinger JM, Snyder AZ, Mintun MA. Increased amygdala response to masked emotional faces in depressed subjects resolves with antidepressant treatment: an fMRI study. Biol Psychiatry 2001;50:651-658.

41. Di Simplicio M, Norbury R, Harmer CJ. Short-term antidepressant administration reduces negative self-referential processing in the medial prefrontal cortex in subjects at risk for depression. Mol Psychiatry 2012;17:503-510.

42. Merz EC, He X, Noble KG. Anxiety, depression, impulsivity, and brain structure in children and adolescents. Neuroimage Clin 2018;20:243-251.

43. Joormann J, Gotlib IH. Selective attention to emotional faces following recovery from depression. J Abnorm Psychol 2007;116:80-85.

44. Duque A, Vazquez C. Double attention bias for positive and negative emotional faces in clinical depression: evidence from an eye-tracking study. J Behav Ther Exp Psychiatry 2015;46:107-114.

45. Platt B, Murphy SE, Lau JY. The association between negative attention biases and symptoms of depression in a community sample of adolescents. Peer J 2015;3:e1372.

46. Wells TT, Beevers CG. Biased attention and dysphoria: Manipulating selective attention reduces subsequent depressive symptoms. Cogn Emot 2010;24:719-728. 


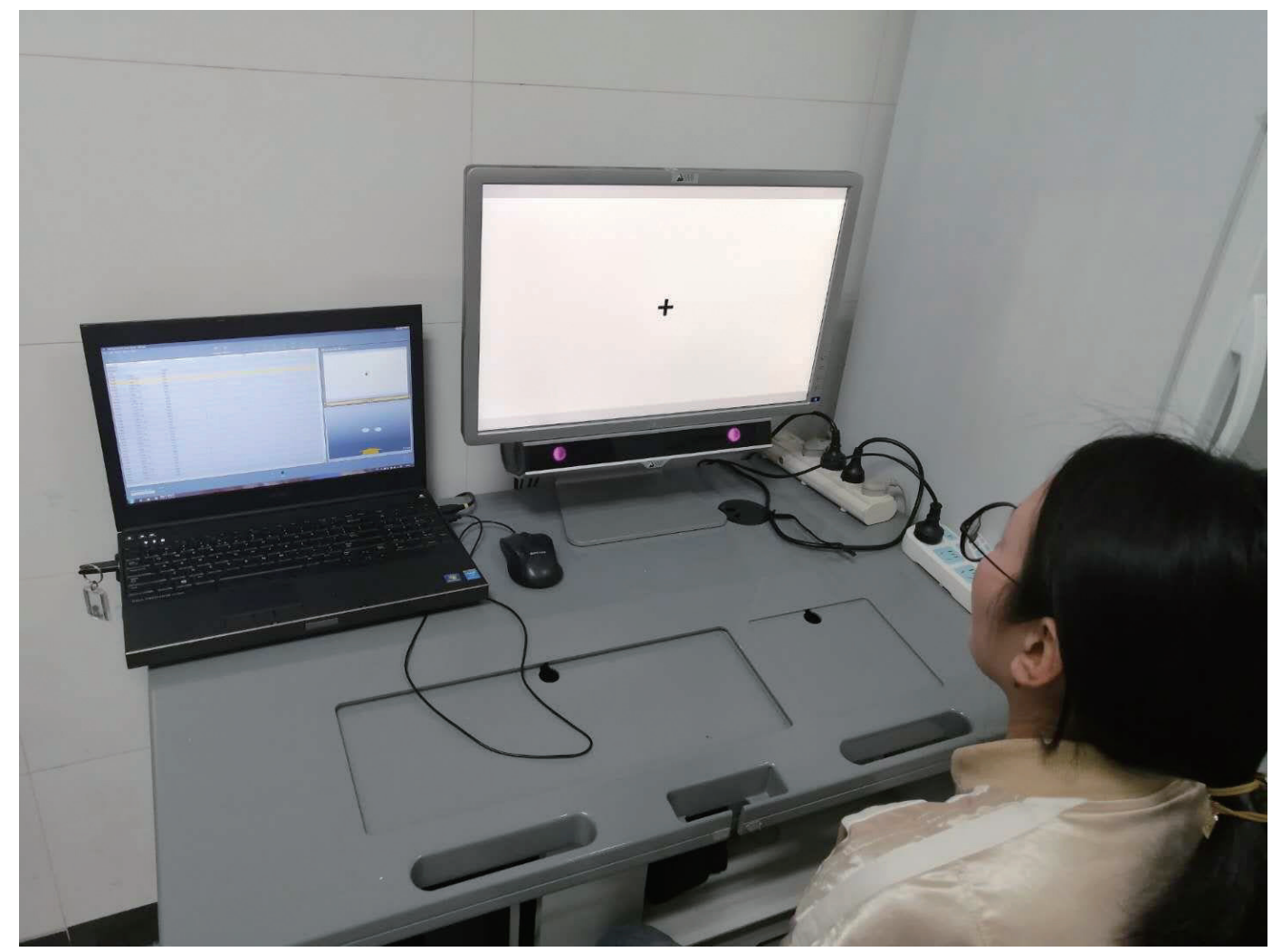

Supplementary Figure 1. The experimental process of eye movement tracking. 


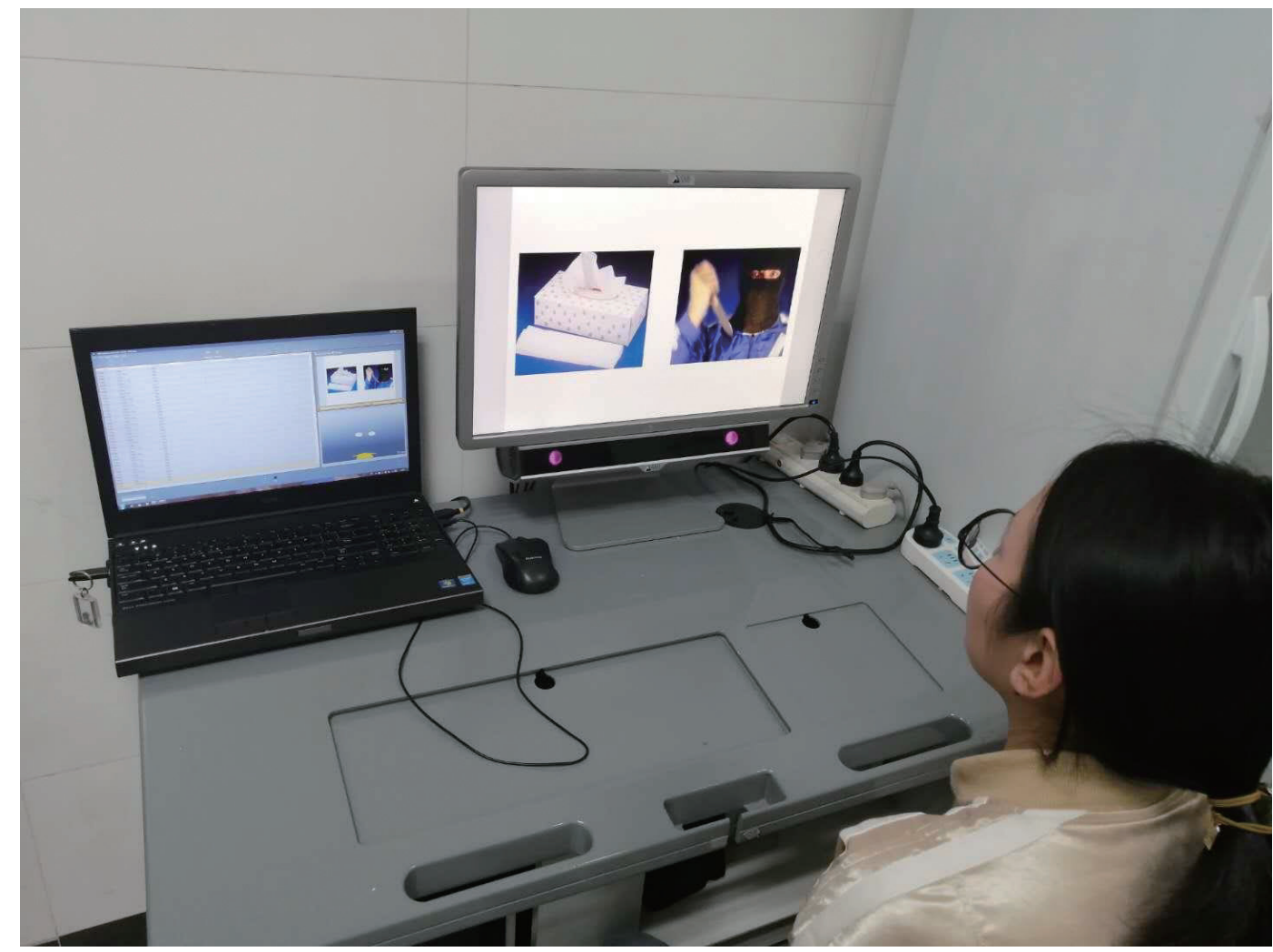

Supplementary Figure 2. The experimental process of eye movement tracking. 